JOURNAL OF INTEGRAL EQUATIONS

AND APPLICATIONS

Volume 11, Number 2, Summer 1999

\title{
TWO-GRID METHODS FOR THE SOLUTION OF NONLINEAR WEAKLY SINGULAR INTEGRAL EQUATIONS BY PIECEWISE POLYNOMIAL COLLOCATION
}

\author{
ENN TAMME
}

\begin{abstract}
To solve nonlinear weakly singular integral equations by the piecewise polynomial collocation method, it is necessary to solve large nonlinear systems. This can be done straightforwardly only for comparatively rough discretizations. In this paper a two-grid iteration method is presented which enables us to find the solution of such systems for fine discretizations. We prove the convergence and establish the convergence rate of this method. So we generalize for nonlinear equations the results proved in [10] for linear equations.
\end{abstract}

1. Introduction. We shall deal with the nonlinear weakly singular integral equation

$$
u(x)=\int_{G} K(x, y, u(y)) d y+f(x), \quad x \in G,
$$

where

$$
G=\left\{x=\left(x_{1}, \ldots, x_{n}\right): 0<x_{k}<b_{k}, k=1, \ldots, n\right\}
$$

is an $n$-dimensional parallelepiped. The piecewise polynomial collocation method for the solution of such equations is considered in $[\mathbf{1}, \mathbf{5}$, $8, \mathbf{1 2}$. In order to calculate the approximate solution by collocation method, large nonlinear systems must be solved. In the present paper a two-grid iteration scheme is presented for the solution of such systems. Fast convergence of this method is shown. Analogous results have been established for linear equations in [10] and for nonlinear equations in the case of piecewise constant collocation method in [9].

Received by the editors on March 17, 1998, and in revised from on September 14, 1998.

Research supported by the Estonian Science Foundation grant No. 2999.

Copyright (C)1999 Rocky Mountain Mathematics Consortium 
2. Integral equation. We shall make the following assumptions (A1)-(A3).

(A1) The kernel $K(x, y, u)$ is $m$ times, $m \geq 1$, continuously differentiable with respect to $x, y$ and $u$ for $x \in G, y \in G, x \neq y, u \in(-\infty, \infty)$, whereby there exists a real number $\nu \in(-\infty, n)$ such that, for any nonnegative integer $l \in \mathbf{Z}_{+}$and multi-indices $\alpha=\left(\alpha_{1}, \ldots, \alpha_{n}\right) \in \mathbf{Z}_{+}^{n}$ and $\beta=\left(\beta_{1}, \ldots, \beta_{n}\right) \in \mathbf{Z}_{+}^{n}$ with $l+|\alpha|+|\beta| \leq m$, the following inequalities hold:

$$
\begin{aligned}
& \left|D_{x}^{\alpha} D_{x+y}^{\beta}\left(\frac{\partial}{\partial u}\right)^{l} K(x, y, u)\right| \\
& \leq \psi_{1}(|u|)\left\{\begin{array}{ll}
1 & \nu+|\alpha|<0 \\
1+|\log | x-y|| & \nu+|\alpha|=0 \\
|x-y|^{-\nu-|\alpha|} & \nu+|\alpha|>0
\end{array},\right. \\
& \left|D_{x}^{\alpha} D_{x+y}^{\beta}\left(\frac{\partial}{\partial u}\right)^{l} K\left(x, y, u_{1}\right)-D_{x}^{\alpha} D_{x+y}^{\beta}\left(\frac{\partial}{\partial u}\right)^{l} K\left(x, y, u_{2}\right)\right| \\
& \leq \psi_{2}\left(\max \left\{\left|u_{1}\right|,\left|u_{2}\right|\right\}\right)\left|u_{1}-u_{2}\right| \begin{cases}1 & \nu+|\alpha|<0 \\
1+|\log | x-y|| & \nu+|\alpha|=0 \\
|x-y|^{-\nu-|\alpha|} & \nu+|\alpha|>0 .\end{cases}
\end{aligned}
$$

Here $|\alpha|=\alpha_{1}+\ldots+\alpha_{n}$ for $\alpha \in \mathbf{Z}_{+}^{n},|x|=\left(x_{1}^{2}+\ldots+x_{n}^{2}\right)^{1 / 2}$ for $x \in \mathbf{R}^{n}$,

$$
\begin{aligned}
D_{x}^{\alpha} & =\left(\frac{\partial}{\partial x_{1}}\right)^{\alpha_{1}} \ldots\left(\frac{\partial}{\partial x_{n}}\right)^{\alpha_{n}}, \\
D_{x+y}^{\beta} & =\left(\frac{\partial}{\partial x_{1}}+\frac{\partial}{\partial y_{1}}\right)^{\beta_{1}} \ldots\left(\frac{\partial}{\partial x_{n}}+\frac{\partial}{\partial y_{n}}\right)^{\beta_{n}},
\end{aligned}
$$

and the functions $\psi_{1}:[0, \infty) \rightarrow[0, \infty)$ and $\psi_{2}:[0, \infty) \rightarrow[0, \infty)$ are assumed to be monotonically increasing.

(A2) The righthand term $f \in C^{m, \nu}(G)$ with the same $m$ and $\nu$ as in (A1), i.e., $f(x)$ is $m$ times continuously differentiable on $G$ and the estimates

$$
\left|D^{\alpha} f(x)\right| \leq \mathrm{const} \begin{cases}1 & |\alpha|<n-\nu \\ 1+|\log \rho(x)| & |\alpha|=n-\nu \\ \rho(x)^{n-\nu-|\alpha|} & |\alpha|>n-\nu\end{cases}
$$




$$
\left|\frac{\partial^{l} f(x)}{\partial x_{k}^{l}}\right| \leq \mathrm{const} \begin{cases}1 & l<n-\nu \\ 1+\left|\log \rho_{k}(x)\right| & l=n-\nu \\ \rho_{k}(x)^{n-\nu-l} & l>n-\nu\end{cases}
$$

hold for $x \in G,|\alpha| \leq m, l=1, \ldots, m, k=1, \ldots, n$, where $\rho_{k}(x)=\min \left\{x_{k}, b_{k}-x_{k}\right\}$ and $\rho(x)=\min _{1 \leq k \leq n} \rho_{k}(x)$ is the distance from $x$ to $\partial G$, the boundary of $G$.

(A3) Integral equation (1) has a solution $u_{0} \in L^{\infty}(G)$ and the linearized integral equation

$$
v(x)=\int_{G} K_{0}(x, y) v(y) d y, \quad K_{0}(x, y)=\left[\frac{\partial K(x, y, u)}{\partial u}\right]_{u=u_{0}(y)},
$$

has only the trivial solution $v=0$ in $L^{\infty}(G)$.

Note that the assumption (A1) holds, for example, for the kernels $K(x, y, u)=K_{1}(x, y, u)|x-y|^{-\nu}, 0<\nu<n$, and $K(x, y, u)=$ $K_{1}(x, y, u) \log |x-y|, \nu=0$, where $K_{1}(x, y, u)$ is an $m+1$ times continuously differentiable function with respect to $x, y, u$ for $x, y \in \bar{G}$, $u \in(-\infty, \infty)$.

From (A1)-(A3) it follows that the solution $u_{0}$ of (1) belongs to the space $C^{m, \nu}(G)[\mathbf{7}, \mathbf{1 2}]$.

3. Collocation method. We use the same non-uniform grid as in $[\mathbf{1 0}, \mathbf{1 2}]$. To define the partition of $\bar{G}$ into cells we choose a vector $N=\left(N_{1}, \ldots, N_{n}\right)$ of natural numbers and introduce in the interval $\left[0, b_{k}\right], k=1, \ldots, n$, the following $2 N_{k}+1$ grid points:

$$
\begin{aligned}
x_{k, N}^{j_{k}} & =\frac{b_{k}}{2}\left(\frac{j_{k}}{N_{k}}\right)^{r}, \quad j_{k}=0,1, \ldots, N_{k}, \\
x_{k, N}^{N_{k}+j_{k}} & =b_{k}-x_{k, N}^{N_{k}-j_{k}}, \quad j_{k}=1, \ldots, N_{k} .
\end{aligned}
$$

Here $r \in \mathbf{R}, r \geq 1$, characterizes the non-uniformity of the grid. If $r=1$, then the grid points (2) are uniformly located. Using the points (2) we introduce the partition of $\bar{G}$ into closed cells $G_{N}^{j}$ :

$$
\begin{gathered}
G_{N}^{j}=\left\{x=\left(x_{1}, \ldots, x_{n}\right): x_{k, N}^{j_{k}-1} \leq x_{k} \leq x_{k, N}^{j_{k}}, k=1, \ldots, n\right\} \subset \bar{G}, \\
j \in J_{N}=\left\{j=\left(j_{1}, \ldots, j_{n}\right): j_{k}=1, \ldots, 2 N_{k}, k=1, \ldots, n\right\} .
\end{gathered}
$$


We determine the collocation points in the following way. We choose $m$ points $\eta_{1}, \ldots, \eta_{m}$ in the interval $[-1,1]$ :

$$
-1 \leq \eta_{1}<\eta_{2}<\cdots<\eta_{m} \leq 1 .
$$

By affine transformations we transfer them into the interval $\left[x_{k, N}^{j_{k}-1}, x_{k, N}^{j_{k}}\right], j_{k}=1, \ldots, 2 N_{k}, k=1, \ldots, n$,

$$
\xi_{k, N}^{j_{k}, q_{k}}=x_{k, N}^{j_{k}-1}+\frac{\eta_{q_{k}}+1}{2}\left(x_{k, N}^{j_{k}}-x_{k, N}^{j_{k}-1}\right), \quad q_{k}=1, \ldots, m .
$$

Note that $\xi_{k, N}^{j_{k}, m}=\xi_{k, N}^{j_{k}+1,1}=x_{k, N}^{j_{k}}$ if $\eta_{1}=-1$ and $\eta_{m}=1, j_{k}=$ $1, \ldots, 2 N_{k}-1$. We assign the collocation points

$$
\begin{gathered}
\xi_{N}^{j, q}=\left(\xi_{1, N}^{j_{1}, q_{1}}, \ldots, \xi_{n, N}^{j_{n}, q_{n}}\right), \quad q \in Q, \\
Q=\left\{q=\left(q_{1}, \ldots, q_{n}\right): q_{k}=1, \ldots, m, k=1, \ldots, n\right\},
\end{gathered}
$$

to the cells $G_{N}^{j}, j \in J_{N}$.

We define the interpolation projector $\mathcal{P}_{N}$ by the formula

$$
\left(\mathcal{P}_{N} u\right)(x)=\sum_{q \in Q} u\left(\xi_{N}^{j, q}\right) \varphi_{N}^{j, q}(x), \quad x \in G_{N}^{j}, j \in J_{N},
$$

where

$$
\varphi_{N}^{j, q}(x)=\varphi_{1, N}^{j_{1}, q_{1}}\left(x_{1}\right) \cdots \varphi_{n, N}^{j_{n}, q_{n}}\left(x_{n}\right)
$$

and $\varphi_{k, N}^{j_{k}, q_{k}}\left(x_{k}\right), k=1, \ldots, n$, are the polynomials of one variable of degree $m-1$ such that

$$
\varphi_{k, N}^{j_{k}, q_{k}}\left(\xi_{k, N}^{j_{k}, p_{k}}\right)=\left\{\begin{array}{ll}
1 & \text { if } p_{k}=q_{k} \\
0 & \text { if } p_{k} \neq q_{k}
\end{array}, \quad p_{k}=1, \ldots, m .\right.
$$

Let us denote by $E_{N}$ the range of the projection $\mathcal{P}_{N}$. This is the finite dimensional space of piecewise polynomial functions $u_{N}$ on $\bar{G}$ which on any cell $G_{N}^{j}, j \in J_{N}$, are polynomials of the degree not exceeding $m-1$ with respect to any of arguments $x_{1}, \ldots, x_{n}$.

We determine the approximate solution $u_{N} \in E_{N}$ of the integral equation (1) by the collocation method from the following conditions:

(5) $\left[u_{N}(x)-\int_{G} K\left(x, y, u_{N}(y)\right) d y-f(x)\right]_{x=\xi_{N}^{i, p}}=0, \quad p \in Q, i \in J_{N}$. 
By the representation (3), we can find $u_{N} \in E_{N}$ in the form

$$
u_{N}(x)=\sum_{q \in Q} c^{j, q} \varphi_{N}^{j, q}(x) \quad \text { if } x \in G_{N}^{j}, j \in J_{N},
$$

where, as it follows from (4),

$$
c^{j, q}=u_{N}\left(\xi_{N}^{j, q}\right)
$$

Now the collocation conditions (5) will take the following form of a nonlinear system which determines the coefficients $c^{j, q}=u_{N}\left(\xi_{N}^{j, q}\right)$ :

$$
\begin{gathered}
u_{N}\left(\xi_{N}^{i, p}\right)=\sum_{j \in J_{N}} \int_{G_{N}^{j}} K\left(\xi_{N}^{i, p}, y, \sum_{q \in Q} u_{N}\left(\xi_{N}^{j, q}\right) \varphi_{N}^{j, q}(y)\right) d y+f\left(\xi_{N}^{i, p}\right), \\
p \in Q, i \in J_{N} .
\end{gathered}
$$

If $\eta_{1}>-1$ or $\eta_{m}<1$, then this system has $(2 m)^{n} N_{1} \ldots N_{n}=\operatorname{dim} E_{N}$ equations and the same number of unknowns. If $\eta_{1}=-1$ and $\eta_{m}=1$, then some collocation points coincide. The number of different collocation points is $\left[2 N_{1}(m-1)+1\right] \ldots\left[2 N_{n}(m-1)+1\right]=\operatorname{dim} E_{N}$ and the system (6) has the same number of equations and unknowns.

In [12] the following result about the convergence of such collocation methods is proved.

Theorem 1. Let the assumptions (A1)-(A3) hold. Then there exist $N_{0}>0$ and $\delta_{0}>0$ such that, for $N_{k} \geq N_{0}, k=1, \ldots, n$, the collocation conditions (5) define a unique approximation $u_{N} \in E_{N}$ to $u_{0}$ satisfying

$$
\sup _{x \in G}\left|u_{N}(x)-u_{0}(x)\right|<\delta_{0} .
$$

The following error estimate holds:

$$
\begin{aligned}
& \max _{j \in J_{N}} \max _{q \in Q}\left|u_{N}\left(\xi_{N}^{j, q}\right)-u_{0}\left(\xi_{N}^{j, g}\right)\right| \\
& \leq \text { const } h_{N}^{m} \text { for } \begin{cases}r>m /(2(n-\nu)) & \text { if } n-\nu \leq 1 \\
r>m /(n-\nu+1) & \text { if } 1<n-\nu \leq m-1 \\
r \geq 1 & \text { if } n-\nu>m-1\end{cases}
\end{aligned}
$$


where

$$
h_{N}=\max _{1 \leq k \leq n} \frac{b_{k}}{N_{k}} .
$$

It is shown in [8] that for special collocation points a more rapid convergence, the superconvergence, takes place.

To apply the collocation method it is necessary to solve the nonlinear system (6). We write this system in the form

$$
\bar{u}_{N}=\mathcal{T}_{N} \bar{u}_{N}+\bar{f}_{N}
$$

where $\bar{u}_{N}=\left(u_{N}\left(\xi_{N}^{j, q}\right)\right)_{j \in J_{N}, q \in Q}, \bar{f}_{N}=\left(f\left(\xi_{N}^{j, q}\right)\right)_{j \in J_{N}, q \in Q}$ are vectors and

$$
\begin{gathered}
\left(\mathcal{T}_{N} \bar{u}_{N}\right)\left(\xi_{N}^{i, p}\right)=\sum_{j \in J_{N}} \int_{G_{N}^{j}} K\left(\xi_{N}^{i, p}, y, \sum_{q \in Q} u_{N}\left(\xi_{N}^{j, q}\right) \varphi_{N}^{j, q}(y)\right) d y \\
i \in J_{N}, p \in Q .
\end{gathered}
$$

Usually the number of equations in (7) is large which makes solving the system directly rather costly if not impossible. An effective method for solving this system is a two-grid iteration method.

4. Two-grid method. In addition to the original grid corresponding to $N=\left(N_{1}, \ldots, N_{n}\right)$, we define another grid, the coarse grid, corresponding to $M=\left(M_{1}, \ldots, M_{n}\right)$ where $M_{k}, k=1, \ldots, n$, are integers such that $N_{k} / M_{k}$ are integers greater than 1 . Then every cell $G_{N}^{j}, j \in J_{N}$, of the original grid is fully contained in some cell $G_{M}^{i}$ of the coarse grid.

For solving the system (7) the following two-grid iteration method is used:

$$
\begin{aligned}
\bar{v}_{N}^{l} & =\mathcal{T}_{N} \bar{u}_{N}^{l}+\bar{f}_{N}, \\
\bar{w}_{M}^{l}-\mathcal{T}_{M} \bar{w}_{M}^{l} & =\mathcal{R}_{N M} \bar{f}_{N}+\mathcal{R}_{N M} \mathcal{T}_{N} \bar{v}_{N}^{l}-\mathcal{T}_{M} \mathcal{R}_{N M} \bar{v}_{N}^{l}, \\
\bar{u}_{N}^{l+1} & =\bar{v}_{N}^{l}+\mathcal{P}_{M N}\left(\bar{w}_{M}^{l}-\mathcal{R}_{N M} \bar{v}_{N}^{l}\right), \quad l=0,1, \ldots,
\end{aligned}
$$


where $\bar{u}_{N}^{0}$ is the initial guess of $\bar{u}_{N}, \mathcal{P}_{M N}: \mathbf{R}^{d_{M}} \rightarrow \mathbf{R}^{d_{N}}\left(d_{N}=\operatorname{dim} E_{N}\right)$ and $\mathcal{R}_{N M}: \mathbf{R}^{d_{N}} \rightarrow \mathbf{R}^{d_{M}}$ are the operators defined by the formulas:

$$
\begin{aligned}
\left(\mathcal{P}_{M N} \bar{w}_{M}^{l}\right)\left(\xi_{N}^{i, p}\right) & =\sum_{q \in Q} w_{M}^{l}\left(\xi_{M}^{j, q}\right) \varphi_{M}^{j, q}\left(\xi_{N}^{i, p}\right) \text { if } \xi_{N}^{i, p} \in G_{M}^{j}, \\
\left(\mathcal{R}_{N M} \bar{f}_{N}\right)\left(\xi_{M}^{i, p}\right) & =f\left(\xi_{M}^{i, p}\right), \\
\left(\mathcal{R}_{N M} \mathcal{T}_{N} \bar{u}_{N}^{l}\right)\left(\xi_{M}^{i, p}\right) & =\sum_{j \in J_{N}} \int_{G_{N}^{j}} K\left(\xi_{M}^{i, p}, y, \sum_{q \in Q} u_{N}^{l}\left(\xi_{N}^{j, q}\right) \varphi_{N}^{j, q}(y)\right) d y \\
\mathcal{R}_{N M} \bar{v}_{N}^{l} & =\mathcal{R}_{N M} \mathcal{T}_{N} \bar{u}_{N}^{l}+\mathcal{R}_{N M} \bar{f}_{N}
\end{aligned}
$$

For linear integral equations, method (8) coincides with the twogrid method in [10]. For nonlinear integral equations similar methods are considered in $[\mathbf{4}, \mathbf{9}]$. Iteration method (8) resembles the two-grid method in [9]. An essentially new idea in the present two-grid method is the restriction operator $\mathcal{R}_{N M}$ as the collocation points of the coarse grid may not coincide with the collocation points of the fine grid.

To apply method (8), it is necessary for every $l$, to solve the nonlinear system in the form

$$
\bar{w}_{M}-\mathcal{T}_{M} \bar{w}_{M}=\bar{g}_{M}
$$

where

$$
\bar{g}_{M}=\mathcal{R}_{N M} \bar{f}_{N}+\mathcal{R}_{N M} \mathcal{T}_{N}\left(\mathcal{T}_{N} \bar{u}_{N}+\bar{f}_{N}\right)-\mathcal{T}_{M} \mathcal{R}_{N M}\left(\mathcal{T}_{N} \bar{u}_{N}+\bar{f}_{N}\right) .
$$

Note that, compared to the system (7) which corresponds to a fine discretization, system (9) corresponds to coarse discretization, and thus the dimension $d_{M}=\operatorname{dim} E_{M}$ of this system is essentially less than the dimension $d_{N}$ of (7). To solve the system (9), one can use some iterative methods, for example, Newton's method with initial guess $\mathcal{R}_{N M} \bar{v}_{N}^{l}$ for $\bar{w}_{M}^{l}$. If $\bar{u}_{N}^{l}$ is a sufficiently good approximation of the solution $\bar{u}_{N, 0}$ of (7), we can use only one step of Newton's method [9]. Note that efficient two-grid methods for solving nonlinear integral equations are obtained using first Newton's method to solve system (7) and second exploiting at each step a coarse grid also $[\mathbf{1}, \mathbf{3}, \mathbf{6}]$.

For the convergence analysis of the two-grid method (8) we use the approach of [2] and consider the iteration method corresponding to (8) in function spaces. 
We write the integral equation (1) in the form

$$
u=\mathcal{T} u+f
$$

where

$$
(\mathcal{T} u)(x)=\int_{G} K(x, y, u(y)) d y .
$$

It is easy to see that the approximate solution $u_{N} \in E_{N}$ of (1), determined from the collocation conditions (5), is the solution of the equation

$$
u_{N}=\mathcal{P}_{N} \mathcal{T} u_{N}+\mathcal{P}_{N} f .
$$

Define the operators $\mathcal{R}_{\infty N}: E_{N} \rightarrow \mathbf{R}^{d_{N}}$ and $\mathcal{P}_{N \infty}: \mathbf{R}^{d_{N}} \rightarrow E_{N}$ by the equalities:

$$
\begin{aligned}
\left(\mathcal{R}_{\infty N} u\right)\left(\xi_{N}^{j, q}\right) & =u\left(\xi_{N}^{j, q}\right), \quad j \in J_{N}, q \in Q, \\
\left(\mathcal{P}_{N \infty} \bar{u}_{N}\right)(x) & =\sum_{q \in Q} u_{N}\left(\xi_{N}^{j, q}\right) \varphi_{N}^{j, q}(x), \quad x \in G_{N}^{j}, j \in J_{N} .
\end{aligned}
$$

These operators define one-to-one correspondence between elements of $E_{N}$ and $\mathbf{R}^{d_{N}}$. From now on we use the operator $\mathcal{R}_{\infty N}$ for all functions defined in the collocation points $\xi_{N}^{j, q}$.

Denote

$$
u_{N}^{l}=\mathcal{P}_{N \infty} \bar{u}_{N}^{l}, \quad v_{N}^{l}=\mathcal{P}_{N \infty} \bar{v}_{N}^{l}, \quad w_{M}^{l}=\mathcal{P}_{M \infty} \bar{w}_{M}^{l} .
$$

Then

$$
\bar{u}_{N}^{l}=\mathcal{R}_{\infty N} u_{N}^{l}, \quad \bar{v}_{N}^{l}=\mathcal{R}_{\infty N} v_{N}^{l}, \quad \bar{w}_{M}^{l}=\mathcal{R}_{\infty M} w_{M}^{l} .
$$

Making use of the identities

$$
\begin{gathered}
\mathcal{R}_{\infty N} \mathcal{P}_{N \infty}=I, \quad \mathcal{P}_{N \infty} \mathcal{R}_{\infty N}=\mathcal{P}_{N} \\
\mathcal{T}_{N}=\mathcal{R}_{\infty N} \mathcal{T}_{N \infty}, \quad \mathcal{P}_{N \infty} \mathcal{P}_{M N}=\mathcal{P}_{M \infty},
\end{gathered}
$$

we rewrite the formulas (8) as follows:

$$
\begin{aligned}
v_{N}^{l} & =\mathcal{P}_{N} \mathcal{T} u_{N}^{l}+\mathcal{P}_{N} f \\
w_{M}^{l}-\mathcal{P}_{M} \mathcal{T} w_{M}^{l} & =\mathcal{P}_{M} f+\mathcal{P}_{M} \mathcal{T} v_{N}^{l}-\mathcal{P}_{M} \mathcal{T} \mathcal{P}_{M}\left(\mathcal{T} u_{N}^{l}+f\right), \\
u_{N}^{l+1} & =v_{N}^{l}+w_{M}^{l}-\mathcal{P}_{M}\left(\mathcal{T} u_{N}^{l}+f\right), \quad l=0,1, \ldots
\end{aligned}
$$


Whereas $u_{N}^{0}=\mathcal{P}_{N \infty} \bar{u}_{N}^{0} \in E_{N}$ we also have $v_{N}^{l} \in E_{N}, w_{M}^{l} \in E_{M} \subset E_{N}$ and $u_{N}^{l+1} \in E_{N}, l=0,1 \ldots$ Therefore the methods (8) and (12) are equivalent. At the same time the method (12) is an iteration method to solve (11).

The equation (9) is equivalent to the equation

$$
w_{M}-\mathcal{P}_{M} \mathcal{T} w_{M}=g_{M}
$$

where $w_{M}=\mathcal{P}_{M \infty} \bar{w}_{M}$ and

$$
g_{M}=\mathcal{P}_{M} f+\mathcal{P}_{M} \mathcal{T} \mathcal{P}_{N}\left(\mathcal{T} u_{N}+f\right)-\mathcal{P}_{M} \mathcal{T} \mathcal{P}_{M}\left(\mathcal{T} u_{N}+f\right)
$$

In the discussion of solvability of equations (11) and (13) we use the following result which is proved in a more general setting, for example, in [11]. We consider the equations (10) and (13) in Banach space $E$ and assume that $\mathcal{T}: E \rightarrow E$ is a nonlinear operator and $\mathcal{P}_{M} \in \mathcal{L}(E, E)$ are linear uniformly bounded operators, i.e., $\left\|\mathcal{P}_{M}\right\| \leq$ const.

Lemma 1. Let the following conditions hold.

(B1) Equation (10) has a solution $u_{0} \in E$ and

$$
\left\|\mathcal{P}_{M} \mathcal{T} u_{0}-\mathcal{T} u_{0}\right\| \rightarrow 0 \quad \text { as } \min _{1 \leq k \leq n} M_{k} \rightarrow \infty .
$$

(B2) There is a positive $\delta$ such that the operator $\mathcal{T}$ is Fréchet differentiable in the ball $S_{\delta}=\left\{u:\left\|u-u_{0}\right\| \leq \delta\right\}$ and for any $\varepsilon>0$ there is a $\delta_{\varepsilon}, 0<\delta_{\varepsilon}<\delta$, such that

$$
\left\|\mathcal{T}^{\prime}(u)-\mathcal{T}^{\prime}\left(u_{0}\right)\right\| \leq \varepsilon \quad \text { whenever } u \in S_{\delta_{\varepsilon}} .
$$

(B3) The derivative $\mathcal{T}^{\prime}\left(u_{0}\right)$ is a compact operator,

$$
\left\|\mathcal{P}_{M} \mathcal{T}^{\prime}\left(u_{0}\right)-\mathcal{T}^{\prime}\left(u_{0}\right)\right\| \longrightarrow 0 \quad \text { as } \min _{1 \leq k \leq n} M_{k} \rightarrow \infty
$$

and the homogeneous equation $v=\mathcal{T}^{\prime}\left(u_{0}\right) v$ has only the trivial solution in $E$. 
(B4) $\left\|g_{M}-f\right\| \rightarrow 0$ as $\min _{1 \leq k \leq n} M_{k} \rightarrow 0$.

Then there exist $M_{0}>0$ and $\delta_{0}, 0<\delta_{0} \leq \delta$, such that for $M_{k} \geq M_{0}, k=1, \ldots, n$, the equation (13) has a unique solution $w_{M, 0}=\left(I-\mathcal{P}_{M} \mathcal{T}\right)^{-1} g_{M}$ in the ball $S_{\delta_{0}}$. Besides $\left\|u_{M, 0}-u_{0}\right\| \rightarrow 0$ if $\min _{1 \leq k \leq n} M_{k} \rightarrow \infty$ with the error estimate

$$
\left\|w_{M, 0}-u_{0}\right\| \leq \mathrm{const}\left\|\left(\mathcal{P}_{M} \mathcal{T} u_{0}-\mathcal{T} u_{0}\right)+\left(g_{M}-f\right)\right\| .
$$

We can consider the method (12) as an iterative method

$$
u_{N}^{l+1}=\Phi u_{N}^{l}, \quad l=0,1, \ldots,
$$

for solving the equation

$$
u=\Phi u \text {. }
$$

To study the convergence of this iterative method, the following wellknown result is used.

Lemma 2. Let equation (15) have a solution $u_{N, 0} \in E$, and let $S_{N, \delta}=\left\{u:\left\|u-u_{N, 0}\right\| \leq \delta\right\}$. If $\Phi: S_{N, \delta} \rightarrow E$ and

$$
\left\|\Phi^{\prime}(u)\right\| \leq q<1 \quad \text { as } u \in S_{N, \delta}
$$

then $u_{N, 0}$ is the unique solution of equation (15) in $S_{N, \delta}$. For every initial guess $u_{N}^{0} \in S_{N, \delta}$ the iterative method (14) converges to $u_{N, 0}$ with the rate

$$
\left\|u_{N}^{l+1}-u_{N, 0}\right\| \leq q\left\|u_{N}^{l}-u_{N, 0}\right\|, \quad l=0,1, \ldots
$$

In the following we take $E=L^{\infty}(G)$ with norm

$$
\|u\|=\sup _{x \in G}|u(x)| .
$$


From assumption (A1) it follows that $\mathcal{T}: L^{\infty}(G) \rightarrow L^{\infty}(G)$ and that the Fréchet derivative

$$
\left(\mathcal{T}^{\prime}(u) \Delta u\right)(x)=\int_{G} \frac{\partial K(x, y, u(y))}{\partial u} \Delta u(y) d y
$$

exists for any $u \in L^{\infty}(G)$. We use the following estimates for studying of the convergence of the two-grid iteration method.

Lemma 3. Let assumptions (A1) and (A2) hold and $u \in\{u:\|u\| \leq$ const\}. Then

$$
\begin{aligned}
&\left\|\mathcal{P}_{N} f-f\right\| \leq \text { const } \varepsilon_{\nu, h_{N}} \\
&\left\|\mathcal{P}_{N} \mathcal{T} u-\mathcal{T} u\right\| \leq \operatorname{const} \varepsilon_{\nu, h_{N}}
\end{aligned}
$$

and

$$
\left\|\mathcal{P}_{N} \mathcal{T}^{\prime}(u)-\mathcal{T}^{\prime}(u)\right\| \leq \text { const } \varepsilon_{\nu, h_{N}}
$$

where

$$
h_{N}=\max _{1 \leq k \leq n} \frac{b_{k}}{N_{k}}
$$

and

$$
\varepsilon_{\nu, h_{N}}= \begin{cases}h_{N} & \nu<n-1 \\ h_{N}\left(1+\left|\log h_{N}\right|\right) & \nu=n-1 \\ h_{N}^{n-\nu} & \nu>n-1\end{cases}
$$

Proof. Let $x \in G_{N}^{j}$. Then

$$
\begin{aligned}
\left(\mathcal{P}_{N} f\right)(x)-f(x) & =\sum_{q \in Q} f\left(\xi_{N}^{j, q}\right) \varphi_{N}^{j, q}(x)-f(x) \\
& =\sum_{q \in Q} \varphi_{N}^{j, q}(x)\left[f\left(\xi_{N}^{j, q}\right)-f(x)\right] .
\end{aligned}
$$

Note that, see $[\mathbf{1 0}]$ and Lemma 2.4 in $[\mathbf{1 2}]$,

$$
\sup _{x \in G_{N}^{j}}\left|f\left(\xi_{N}^{j, q}\right)-f(x)\right| \leq \text { const } \varepsilon_{\nu, h_{N}}
$$


and

$$
\max _{x \in G_{N}^{j}}\left|\varphi_{N}^{j, q}(x)\right| \leq c^{n}
$$

where

$$
c=\max _{1 \leq q_{k} \leq m} \max _{-1 \leq \eta \leq 1}\left|\prod_{\substack{s=1 \\ s \neq q_{k}}}^{m} \frac{\eta-\eta_{s}}{\eta_{q_{k}}-\eta_{s}}\right| .
$$

Thus

$$
\sup _{x \in G_{N}^{j}}\left|\left(\mathcal{P}_{N} f\right)(x)-f(x)\right| \leq \text { const } \varepsilon_{\nu, h_{N}}, \quad j \in J_{N},
$$

from which follows the estimate (16). The proof of (17) is analogous using a generalization of Lemma 2.3 in [12] for nonlinear operators. The estimate (18) follows from the lemma in [10].

Remark. From (19) it follows that $\left\|\mathcal{P}_{N}\right\| \leq c^{n}=$ const.

We are now ready to prove the following result about the convergence of the two-grid method (8).

Theorem 2. Let the assumptions (A1)-(A3) hold. Then there exist $M_{0}>0$ and $\delta_{0}>0$ such that, for $N_{k} \geq M_{0}, k=1, \ldots, n$, the system (7) has a unique solution $\bar{u}_{N, 0}$ satisfying $\left\|\mathcal{P}_{N \infty} \bar{u}_{N, 0}-u_{0}\right\| \leq \delta_{0}$. The two-grid iteration method (8) converges for $M_{k} \geq M_{0}, k=1, \ldots, n$, and for sufficiently good initial guess $\bar{u}_{N}^{0}$ to this solution with the rate

$$
\left\|\bar{u}_{N}^{l+1}-\bar{u}_{N, 0}\right\| \leq c_{1} \varepsilon_{\nu, h_{M}}\left\|\bar{u}_{N}^{l}-\bar{u}_{N, 0}\right\|, \quad l=0,1, \ldots
$$

where

$$
\left\|\bar{u}_{N}\right\|=\max _{j \in J_{N}} \max _{q \in Q}\left|u_{N}\left(\xi_{N}^{j, q}\right)\right|
$$

Proof. We get by Lemma 3 that the conditions (B1)-(B3) of Lemma 1 are fulfilled with $E=L^{\infty}(G)$. On the grounds of Lemma 1 there exist $M_{0}^{\prime}>0$ and $\delta_{0}>0$ such that for $N_{k} \geq M_{0}^{\prime}, k=1, \ldots, n$, equation (11) has a unique solution $u_{N, 0}$ in the ball $S_{\delta_{0}}$. From this the first assertion of the theorem follows because $u_{N, 0} \in E_{N}$ and $\bar{u}_{N, 0}=\mathcal{R}_{\infty N} u_{N, 0}$ is the solution of (7). 
Method (12) is an iterative method of the form (14) where

$$
\begin{aligned}
\Phi u=\left(\mathcal{P}_{N}-\mathcal{P}_{M}\right)(\mathcal{T} u & +f)+\left(I-\mathcal{P}_{M} \mathcal{T}\right)^{-1}\left[\mathcal{P}_{M} f\right. \\
& \left.+\mathcal{P}_{M} \mathcal{T} \mathcal{P}_{N}(\mathcal{T} u+f)-\mathcal{P}_{M} \mathcal{T} \mathcal{P}_{M}(\mathcal{T} u+f)\right] .
\end{aligned}
$$

It is easy to check that the solution $u_{N, 0}$ of equation (11) is the solution of equation (15) too. The derivative of $\Phi$ is expressed

$$
\begin{aligned}
\Phi^{\prime}(u) \Delta u= & \left(\mathcal{P}_{N}-\mathcal{P}_{M}\right) \mathcal{T}^{\prime}(u) \Delta u \\
+\left[I-\mathcal{P}_{M} \mathcal{T}^{\prime}(w)\right]^{-1}\left[\mathcal{P}_{M} \mathcal{T}^{\prime}\left(\mathcal{P}_{N}(\mathcal{T} u+f)\right) \mathcal{P}_{N}\right. & \left.\quad-\mathcal{P}_{M} \mathcal{T}^{\prime}\left(\mathcal{P}_{M}(\mathcal{T} u+f)\right) \mathcal{P}_{M}\right] \mathcal{T}^{\prime}(u) \Delta u
\end{aligned}
$$

where $w$ is the solution of the equation

$$
w-\mathcal{P}_{M} \mathcal{T} w=g
$$

with

$$
g=\mathcal{P}_{M} f+\mathcal{P}_{M} \mathcal{T} \mathcal{P}_{N}(\mathcal{T} u+f)-\mathcal{P}_{M} \mathcal{T} \mathcal{P}_{M}(\mathcal{T} u+f) .
$$

Denote $v_{N}=\mathcal{P}_{N}(\mathcal{T} u+f)$. Then

$$
g=\mathcal{P}_{M} f+\mathcal{P}_{M} \mathcal{T} v_{N}-\mathcal{P}_{M} \mathcal{T} v_{M}
$$

Let $\|u\| \leq$ const. Then by Lemma 3 we get

$$
\begin{aligned}
\|g-f\| & \leq\left\|\mathcal{P}_{M} f-f\right\|+\left\|\mathcal{P}_{M} \mathcal{T} v_{N}-\mathcal{P}_{M} \mathcal{T} v_{M}\right\| \\
& \leq \operatorname{const} \varepsilon_{\nu, h_{M}}+\text { const }\left\|v_{N}-v_{M}\right\| \leq \operatorname{const} \varepsilon_{\nu, h_{M}} .
\end{aligned}
$$

On the grounds of Lemma 1 there exists $M_{0}^{\prime \prime} \geq M_{0}^{\prime}$ such that for $M_{k} \geq M_{0}^{\prime \prime}, k=1, \ldots, n$, equation (21) has a unique solution $w$ in the ball $S_{\delta_{0}}$ and

$$
\left\|w-u_{0}\right\| \leq \operatorname{const} \varepsilon_{\nu, h_{M}} \rightarrow 0 \text { as } \min _{1 \leq k \leq n} M_{k} \rightarrow \infty .
$$

Analogously we get that the formulas (12) and (8) define sequences $u_{N}^{l} \in E_{N}$ and $\bar{u}_{N}^{l}=\mathcal{R}_{\infty N} u_{N}^{l}, l=1,2, \ldots$, for $M_{k} \geq M_{0}^{\prime \prime}, k=1, \ldots, n$, and for sufficiently good initial guess $u_{N}^{0} \in E_{N}$ to $u_{N, 0}$. 
Due to (A1) and (A3) there exists the inverse operator $\left[I-\mathcal{T}^{\prime}\left(u_{0}\right)\right]^{-1}$. By Lemma 3 and (22) we get

$$
\begin{aligned}
\left\|\mathcal{P}_{M} \mathcal{T}^{\prime}(w)-\mathcal{T}^{\prime}\left(u_{0}\right)\right\| \leq & \left\|\mathcal{P}_{M} \mathcal{T}^{\prime}(w)-\mathcal{P}_{M} \mathcal{T}^{\prime}\left(u_{0}\right)\right\| \\
& +\left\|\mathcal{P}_{M} \mathcal{T}^{\prime}\left(u_{0}\right)-\mathcal{T}^{\prime}\left(u_{0}\right)\right\| \leq \operatorname{const} \varepsilon_{\nu, h_{M}} \longrightarrow 0 \\
& \text { as } \min _{1 \leq k \leq n} M_{k} \rightarrow \infty
\end{aligned}
$$

Therefore we can find $M_{0}^{\prime \prime \prime} \geq M_{0}^{\prime \prime}$ so that for $M_{k} \geq M_{0}^{\prime \prime \prime}, k=1, \ldots, n$, there exist the inverse operators $\left[I-\mathcal{P}_{M} \mathcal{T}^{\prime}(w)\right]^{-1}$ which are uniformly bounded:

$$
\left\|\left[I-\mathcal{P}_{M} \mathcal{T}^{\prime}(w)\right]^{-1}\right\| \leq \text { const. }
$$

For $M_{k} \geq M_{0}^{\prime \prime \prime}, k=1, \ldots, n$, we can estimate

$$
\begin{aligned}
\left\|\Phi^{\prime}(u)\right\| \leq & \left\|\left(\mathcal{P}_{N}-\mathcal{P}_{M}\right) \mathcal{T}^{\prime}(u)\right\| \\
& + \text { const }\left\|\mathcal{P}_{M} \mathcal{T}^{\prime}\left(v_{N}\right) \mathcal{P}_{N} \mathcal{T}^{\prime}(u)-\mathcal{P}_{M} \mathcal{T}^{\prime}\left(v_{M}\right) \mathcal{P}_{M} \mathcal{T}^{\prime}(u)\right\| \\
\leq & \operatorname{const}\left[\varepsilon_{\nu, h_{M}}+\left\|\mathcal{P}_{M}\left[\mathcal{T}^{\prime}\left(v_{N}\right)-\mathcal{T}^{\prime}\left(v_{M}\right)\right] \mathcal{P}_{N} \mathcal{T}^{\prime}(u)\right\|\right. \\
& \left.\quad+\left\|\mathcal{P}_{M} \mathcal{T}^{\prime}\left(v_{M}\right)\left(\mathcal{P}_{N}-\mathcal{P}_{M}\right) \mathcal{T}^{\prime}(u)\right\|\right] \\
\leq & c_{0} \varepsilon_{\nu, h_{M}} \quad \text { if } u \in S_{N, \delta}
\end{aligned}
$$

where $\delta>0$ and $S_{N, \delta} \subset S_{\delta_{0}}$. We choose $M_{0} \geq M_{0}^{\prime \prime \prime}$ so that $c_{0} \varepsilon_{\nu, h_{M}}<1$ as $M_{k} \geq M_{0}, k=1, \ldots, n$. Then from Lemma 2 it follows that the iteration method (12) converges, for $x_{N}^{0} \in S_{N, \delta}$ and $M_{k} \geq M_{0}$, $k=1, \ldots, n$, and

$$
\left\|u_{N}^{l+1}-u_{N, 0}\right\| \leq c_{0} \varepsilon_{\nu, h_{M}}\left\|u_{N}^{l}-u_{N, 0}\right\|, \quad l=1,2, \ldots .
$$

The estimate (20) with $c_{1}=c^{n} c_{0}$ follows from (23) because

$$
\left\|\bar{u}_{N}^{l+1}-\bar{u}_{N, 0}\right\|=\left\|\mathcal{R}_{\infty N}\left(u_{N}^{l+1}-u_{N, 0}\right)\right\| \leq\left\|u_{N}^{l+1}-u_{N, 0}\right\|
$$

and

$$
\left\|u_{N}^{l}-u_{N, 0}\right\|=\left\|\mathcal{P}_{N \infty}\left(\bar{u}_{N}^{l}-\bar{u}_{N, 0}\right)\right\| \leq c^{n}\left\|\bar{u}_{N}^{l}-\bar{u}_{N, 0}\right\| .
$$

The last inequality is a consequence of (19). Theorem 2 is proved. 
From the estimate (20) we see that the two-grid iteration method (8) converges quite quickly provided that $M_{k}, k=1, \ldots, n$, are chosen sufficiently large. Thus we get for the nonlinear equations the same rate of convergence as is proved for linear equations in [10]. In [9] a two-grid iteration method for the solution of nonlinear weakly singular integral equations on arbitrary bounded domain $G$ by piecewise constant collocation method is considered. In the special case when the domain $G$ and the cells $G_{N}^{j}$ are parallelepipeds the main result of [9] follows from Theorem 2 proved above.

Example. Consider the integral equation

$$
u(x)=\int_{0}^{1}|x-y|^{-1 / 2} u^{2}(y) d y+f(x), \quad 0<x<1,
$$

where $f(x)$ is selected so that $u_{0}(x)=\sqrt{x(1-x)}$ is the solution to be approximated, see [8]. It is easy to see that the assumptions (A1)-(A3) hold for this equation with $\nu=1 / 2$. Tables $1-4$ present the norm $\varepsilon_{N}$ of the errors of the approximate solutions $\bar{u}_{N}$ calculated by the collocation method:

$$
\varepsilon_{N}=\left\|\bar{u}_{N}-\bar{u}_{0}\right\|=\max _{j \in J_{N}, q \in Q}\left|u_{N}\left(\xi_{N}^{j, q}\right)-u_{0}\left(\xi_{N}^{j, q}\right)\right| .
$$

TABLE 1.

\begin{tabular}{|r|c|c|c|c|}
\hline & \multicolumn{2}{|c|}{$r=1$} & \multicolumn{2}{c|}{$r=2$} \\
\hline$N$ & $\varepsilon_{N}$ & $\rho_{N}$ & $\varepsilon_{N}$ & $\rho_{N}$ \\
2 & $1.0 \mathrm{E}-1$ & & $8.7 \mathrm{E}-2$ & \\
4 & $3.0 \mathrm{E}-2$ & 3.4 & $2.2 \mathrm{E}-2$ & 4.0 \\
8 & $8.7 \mathrm{E}-3$ & 3.4 & $5.3 \mathrm{E}-3$ & 4.1 \\
16 & $2.4 \mathrm{E}-3$ & 3.6 & $1.3 \mathrm{E}-3$ & 4.0 \\
32 & $6.8 \mathrm{E}-4$ & 3.6 & $3.3 \mathrm{E}-4$ & 4.0 \\
64 & $2.5 \mathrm{E}-4$ & 2.8 & $8.2 \mathrm{E}-5$ & 4.0 \\
128 & $8.8 \mathrm{E}-5$ & 2.8 & $2.0 \mathrm{E}-5$ & 4.0 \\
\hline
\end{tabular}


TABLE 2 .

\begin{tabular}{|r|r|c|c|r|c|}
\hline & & \multicolumn{2}{|c|}{$r=1$} & \multicolumn{2}{c|}{$r=2$} \\
\hline$M$ & $N$ & $l_{0}$ & $\varepsilon_{N}$ & $l_{0}$ & $\varepsilon_{N}$ \\
\hline 4 & 8 & 4 & $8.7 \mathrm{E}-3$ & 4 & $5.5 \mathrm{E}-3$ \\
\hline 4 & 16 & 5 & $2.5 \mathrm{E}-3$ & 5 & $1.4 \mathrm{E}-3$ \\
8 & 16 & 3 & $2.4 \mathrm{E}-3$ & 3 & $1.3 \mathrm{E}-3$ \\
\hline 4 & 64 & 6 & $2.5 \mathrm{E}-4$ & 8 & $8.2 \mathrm{E}-5$ \\
8 & 64 & 4 & $2.5 \mathrm{E}-4$ & 5 & $8.0 \mathrm{E}-5$ \\
16 & 64 & 3 & $2.5 \mathrm{E}-4$ & 3 & $8.3 \mathrm{E}-5$ \\
32 & 64 & 3 & $2.5 \mathrm{E}-4$ & 2 & $8.3 \mathrm{E}-5$ \\
\hline 4 & 256 & 8 & $3.2 \mathrm{E}-5$ & 12 & $4.9 \mathrm{E}-6$ \\
8 & 256 & 5 & $3.1 \mathrm{E}-5$ & 8 & $5.1 \mathrm{E}-6$ \\
16 & 256 & 3 & $3.2 \mathrm{E}-5$ & 5 & $5.1 \mathrm{E}-6$ \\
32 & 256 & 3 & $3.1 \mathrm{E}-5$ & 4 & $5.1 \mathrm{E}-6$ \\
64 & 256 & 3 & $3.1 \mathrm{E}-5$ & 3 & $5.1 \mathrm{E}-6$ \\
\hline
\end{tabular}

TABLE 3.

\begin{tabular}{|r|c|c|c|c|r|r|}
\hline & \multicolumn{2}{|c|}{$r=1$} & \multicolumn{2}{c|}{$r=2$} & \multicolumn{2}{c|}{$r=4$} \\
\hline$N$ & $\varepsilon_{N}$ & $\rho_{N}$ & $\varepsilon_{N}$ & $\rho_{N}$ & $\varepsilon_{N}$ & $\rho_{N}$ \\
\hline 2 & $7.8 \mathrm{E}-4$ & & $1.9 \mathrm{E}-3$ & & $6.7 \mathrm{E}-3$ & \\
4 & $1.9 \mathrm{E}-4$ & 4.0 & $2.2 \mathrm{E}-4$ & 8.9 & $1.5 \mathrm{E}-3$ & 4.5 \\
8 & $7.0 \mathrm{E}-5$ & 2.8 & $3.4 \mathrm{E}-5$ & 6.5 & $2.1 \mathrm{E}-4$ & 7.0 \\
16 & $2.3 \mathrm{E}-5$ & 3.0 & $5.2 \mathrm{E}-6$ & 6.5 & $2.7 \mathrm{E}-5$ & 8.0 \\
32 & $7.7 \mathrm{E}-6$ & 3.0 & $1.0 \mathrm{E}-6$ & 5.2 & $6.7 \mathrm{E}-6$ & 4.0 \\
64 & $2.6 \mathrm{E}-6$ & 2.9 & $1.7 \mathrm{E}-7$ & 5.9 & $1.4 \mathrm{E}-6$ & 4.8 \\
\hline
\end{tabular}

The results of Tables 1 and 3 have been obtained by the solution of the nonlinear system (6) with Newton's method and of Tables 2 and 4 by the solution of this system with the two-grid method (8) using $l_{0}$ iterative steps. The number of steps $l_{0}$ is chosen so that the errors in Tables 2 and 4 are within $3 \%$ at the error of the corresponding 
TABLE 4

\begin{tabular}{|r|r|r|c|r|c|r|c|}
\hline & & \multicolumn{2}{|c|}{$r=1$} & \multicolumn{2}{c|}{$r=2$} & \multicolumn{2}{c|}{$r=4$} \\
\hline$M$ & $N$ & $l_{0}$ & $\varepsilon_{N}$ & $l_{0}$ & $\varepsilon_{N}$ & $l_{0}$ & $\varepsilon_{N}$ \\
\hline 4 & 8 & 4 & $6.9 \mathrm{E}-5$ & 11 & $3.4 \mathrm{E}-5$ & 13 & 2.2 E-4 \\
\hline 4 & 16 & 4 & 2.4 E-5 & 11 & 5.1 E-6 & 12 & 2.6 E-5 \\
8 & 16 & 3 & 2.3 E-5 & 7 & 5.1 E-6 & 11 & 2.7 E-5 \\
\hline 4 & 32 & 6 & 7.9 E-6 & 13 & 1.0 E-6 & 13 & 6.8 E-6 \\
8 & 32 & 3 & 7.7 E-6 & 8 & 1.0 E-6 & 10 & 6.3 E-6 \\
16 & 32 & 3 & 7.7 E-6 & 4 & 1.0 E-6 & 6 & 6.5 E-6 \\
\hline 4 & 64 & 7 & 2.7 E-6 & 16 & 1.7 E-7 & 18 & 1.3 E-6 \\
8 & 64 & 4 & 2.7 E-6 & 10 & 1.7 E-7 & 12 & 1.3 E-6 \\
16 & 64 & 2 & 2.5 E-6 & 7 & 1.7 E-7 & 8 & 1.4 E-6 \\
32 & 64 & 2 & 2.6 E-6 & 4 & 1.7 E-7 & 3 & 1.4 E-6 \\
\hline
\end{tabular}

collocation method. All the integrals needed for the construction of the system (6) were found analytically.

We have chosen $m=2$ and $-\eta_{1}=\eta_{2}=1$ in the case of Tables 1 and 2 and $-\eta_{1}=\eta_{2}=1 / \sqrt{3}$ in the case of Tables 3 and 4 (the last values of $\eta_{1}$ and $\eta_{2}$ determine the collocation points of the superconvergence). The number of the equations and unknowns in system (6) is in the first case $2 N+1$ and in the second case $4 N$. The ratio $\rho_{N}=\varepsilon_{N / 2} / \varepsilon_{N}$ characterizes the rate of the convergence of the collocation method. It is proved $[\mathbf{8}, \mathbf{1 2}]$ that the error of the collocation method $\varepsilon_{N}=O\left(N^{-2}\right)$ in the first case for $r>2$ and $\varepsilon_{N}=O\left(N^{-5 / 2}\right)$ in the second case for $r>4$. Thus the value of the ratio $\rho_{N}$ ought to be respectively $2^{2}=4$ and $2^{5 / 2} \approx 5.7$. From Tables 1 and 3 we see that such rate of convergence is achieved already for $r=2$. For $r=4$ the errors of the collocation method are bigger and the rate of the convergence of the two-grid iteration method is slower than for $r=2$.

The initial guess of the two-grid method has been taken $\bar{u}_{N}^{0}=$ $\mathcal{P}_{M N} \bar{u}_{M}$ in the case of Table 2 and $\bar{u}_{N}^{0}=\mathcal{R}_{M N} \mathcal{T}_{M} \bar{u}_{M}+\bar{f}_{N}$ in the case of Table 4 . In the case of Table 4 one can also use the initial guess $\bar{u}_{N}^{0}=\mathcal{P}_{M N} \bar{u}_{M}$ but then in most cases we need one extra approximation 
step of the two-grid method to get the same precision. Such initial guesses are so good that it is sufficient to make only one step of Newton's method with initial guess $\mathcal{R}_{N M} v_{N}^{l}$ for $w_{M}^{l}$ for the solution of the system (9).

From numerical examples it follows that the estimate (20) of Theorem 2 expresses quite well the convergence rate of the two-grid method. This method converges approximately with the rate of the geometric progression, which factor essentially behaves like const $M^{-1 / 2}$. It appears that one can use rather coarse grids even for a quite fine initial grid. A good strategy will be $M \approx \sqrt{N}$.

\section{REFERENCES}

1. K.E. Atkinson, A survey of numerical methods for solving nonlinear integral equations, J. Integral Equations Appl. 4 (1992), 15-46.

2. - Two-grid iteration methods for linear integral equations of the second kind on piecewise smooth surfaces in $\mathbf{R}^{3}$, SIAM J. Sci. Comput. 15 (1994), 1083-1104.

3. - The numerical solution of a non-linear boundary integral equation on smooth surfaces, IMA J. Numer. Anal. 14 (1994), 461-483.

4. W. Hackbusch, Multi-grid methods and applications, Springer-Verlag, Berlin, 1985.

5. H. Kaneko, R. Noren and Y. Xu, Numerical solutions for weakly singular Hammerstein equations and their superconvergence, J. Integral Equations Appl. 4 (1992), 391-407.

6. C.T. Kelley, A fast multilevel algorithm for integral equations, SIAM J. Numer. Anal. 32 (1995), 501-513.

7. A. Pedas and G. Vainikko, Tangential derivatives of solutions to nonlinear multidimensional weakly singular integral equations, in Beiträge zur angewandten Analysis und Informatik, Shaker Verlag, Aachen, 1994, 271-287.

8. - Superconvergence of piecewise polynomial collocations for nonlinear weakly singular integral equations, J. Integral Equations Appl. 9 (1997), 379-406.

9. E. Tamme, Two-grid method for nonlinear multi-dimensional weakly singular integral equations, J. Integral Equations Appl. 7 (1995), 99-113.

10. - Two-grid method for the solution of weakly singular integral equations by piecewise polynomial approximation, Proc. Estonian Acad. Sci. Phys. Math. 46 (1997), 241-250.

11. G. Vainikko, Approximative methods for nonlinear equations, Nonlin. Analysis 2 (1978), 647-687. 
12. - Multidimensional weakly singular integral equations, Lecture Notes in Math. 1549 (1993).

Institute of Applied Mathematics, University of Tartu, Lini Str. 2 206, 50409 TARTU, Estonia.

E-mail address: enn.tamme@ut.ee 